\title{
SPECTROSCOPIC AND THERMOPHYSICAL INVESTIGATIONS OF NEW SYNTHESIZED AZO DYE AND ITS APPLICATION IN POLARIZING FILM
}

\author{
Siyamak SHAHAB,${ }^{\mathrm{a}, \mathrm{b}, \mathrm{c}, *}$ Liudmila FILIPPOVICH,${ }^{\mathrm{a}, \mathrm{b}}$ Masoome SHEIKHI, ${ }^{\mathrm{d}, *}$ Maria AHARODNIKOVA ${ }^{\mathrm{a}}$ \\ and Rakesh KUMAR ${ }^{\mathrm{e}}$

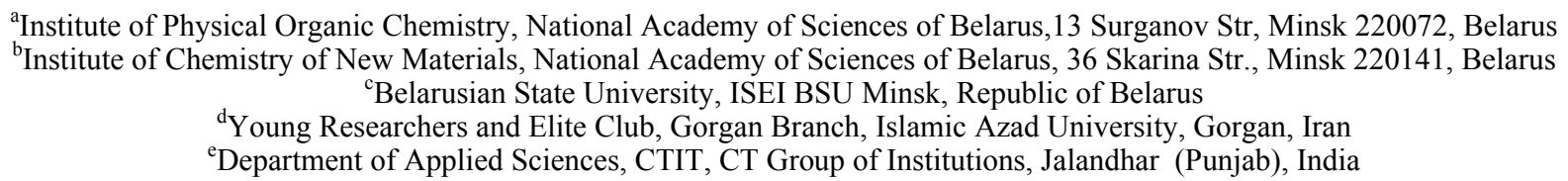

Received March 27, 2017

Quantum-chemical calculations using the Density Functional Theory (DFT) approach for structural analysis of a new azo benzene derivative: Sodium 6-amino-5-hydroxy-3-((E)-(2methoxy-4-((E)-(4-sulfonatophenyl)diazenyl)phenyl)diazenyl)naphthalene-2-sulfonate (A) (trans isomer) were designed using B3LYP method with $6-311++\mathrm{G}^{* *}$ basis set. The electronic absorption spectrum of the $\mathbf{A}$ in water solution was calculated using TDB3LYP/6$311++\mathrm{G}^{* *}$ method. The excitation energies, electronic transitions and oscillator strengths for studied structure have also been calculated. On the basis of calculations a new dichroic azo benzene dye A was synthesized. The experimental UV-Vis, FT-IR and ${ }^{1} \mathrm{H}$ NMR spectra of the title molecule were presented. On the basis of polyvinyl alcohol (PVA) and the dichroic synthesized azo benzene dye A thermostable polarizing film absorbing in the seen region of the spectrum $\left(\lambda_{\max }=550 \mathrm{~nm}\right)$ with Polarization Efficiency $(\mathrm{PE})$ in the absorption maximum $\left(\lambda_{\max }=92 \%\right)$ and Stretching Degree $\left(\mathrm{R}_{\mathrm{s}}\right)=3.0$ was developed. The experimental spectral-polarization parameters of stretched colored PVA-films were measured. During the work it was established that oriented PVA-films is phenomenon of anisotropy of thermal conductivity $\left(\lambda_{\|} / \lambda_{\perp}\right)$. Thermal conductivity in a direction of orientation $\left(\lambda_{\|}\right)$is higher than in a direction perpendicular orientations $\left(\lambda_{\perp}\right)$.

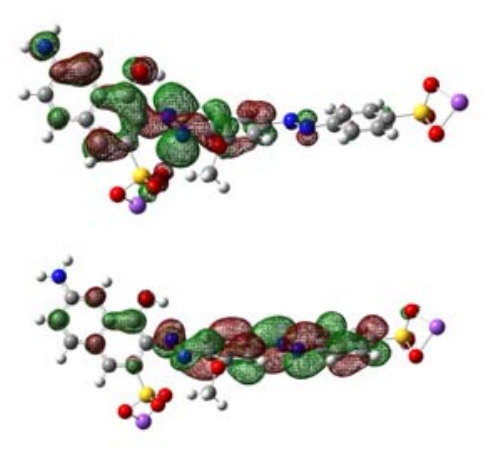

\section{INTRODUCTION}

The polarizing thin film presents a uniaxial oriented polymeric film containing dichroic agent. Light-polarizing films are the basis of sheet polarizers which are traditionally used in processes of manufacturing of liquid crystal indicators for displaying devices. They are constituted usually from polymer matrix, e.g., polyethylene or polyvinyl alcohol containing uniaxial oriented dichroic dyes or iodine. ${ }^{1,2}$ The most used polarizers are the $\mathrm{H}$-type polarizers which contain molecular iodine as a dichroic agent. They are excellent in the initial polarization performance but are weak against water or heat. L-type polarizers contain a dichroic dye or mixture of the dyes as a polarizing agent. ${ }^{3,4}$ Examples of dichroic molecules include dye compounds, such as azo dyes or as well as iodine have a high polarization degree (90-99\%) and can be maintained in a wide range of temperatures. Dichroic dyes when employed as polarizing films are generally used in combination with other dyes, having absorption in a particular wavelength range to provide the polarizing films with a neutral color and wide range spectra. ${ }^{5,6}$ PVA-films are easily exposed to uniaxial orientation. This

\footnotetext{
${ }^{*}$ Corresponding authors: siyamak.shahab@yahoo.com (S. Shahab); m.sheikhi2@gmail.com (M. Sheikhi)
} 
property of PVA has defined theoretical and practical interest in it from the point of view of their use for the manufacturing of film polarizers. The majority of films with dichroic dyes finding practical application polarize light in a visible area $(400-700 \mathrm{~nm})$ electromagnetic spectrum where the basic strips of absorption of molecules with stretched chains of the interfaced double bond conjugation are located. Theoretical quantum chemistry methods based on HF and DFT are used for the calculation of optimized geometry, absorption spectrum, FT-IR and ${ }^{\mathrm{P}} \mathrm{H}$ NMR spectra of the organic molecules. ${ }^{5-17}$ In this work on the basis of PVA and new synthesized dichroic azo benzene dye $\mathbf{A}$ thermostable polarizing film absorbing in visible spectral range of spectrum $\left(\lambda_{\max }\right.$ $=550 \mathrm{~nm}$ ) for application in Display Technologies and Optoelectronic applications was developed.

\section{EXPERIMENTAL}

\section{Reagent and apparatus}

All chemicals used were of analytical reagent grade. PVA used in this work «Mowiol 28-99») was purchased from (Hoëchst Akiengesllschaft Co., Germany). The experimental UV/Vis absorption spectrum of the molecule was recorded on UV-Visible Spectrophotometer Cary 300 (Varian, USA). The optical transmission spectra were measured in polarized light with UVNIR Spectrophotometer HR 4000 (Ocean optics, USA). FT-IR spectrum of films was measured by a spectrophotometer of
Protégé 460 (Nicolet, US). The experimental FT-IR spectrum was recorded by KBr pellet method with spectral resolution $2 \mathrm{~cm}^{-1} .{ }^{1} \mathrm{H}$ NMR spectrum was recorded in DMSO- $\mathrm{d}_{6}$ by NMR AVANCE500 spectrometer (Bruker, Germany) with a working frequency of $600 \mathrm{~Hz}(1 \mathrm{H})$. During calibration of the chemical shifts as the internal standard the residual signal of the solvent was used. Thermal conductivity of films was measured on the complex equipment LC - 201 (Alfa Laval Group, Sweden) using indicator method for the determination of heat conductivity of polymer materials and films.

Synthesis of Sodium 6-amino-5-hydroxy-3-((E)-(2-methoxy-4((E)-(4-sulfonato-

phenyl)diazenyl)phenyl)diazenyl)naphthalene-2-sulfonate (A)

Azo coupling diazotized sulfanilic acid with $o$-anisidine was carried out in formic acid (I). Thus was obtained 4-(4-amino-3methoxyphenylazo)-benzolsulfonic acid as a chromatographically pure product (58\% yield), which was diazotized and treated with $\gamma$-acid in an alkaline medium by the classical method (II). The diazo compound was isolated by treating the reaction mixture with sodium chloride. The yield after recrystallization from $70 \%$ ethanol was $79.4 \%$. The dye was dried in vacuum at $10 \mathrm{~mm} \mathrm{Hg}$ at $150^{\circ} \mathrm{C}$. The final product are dark violet crystals (III) (Fig. 1). This used method avoids the formation of triazenes and products of their transformation.

\section{Experimental ${ }^{1} H$ NMR Spectrum of the dye $A \delta$}

(DMSO-d6, ppm) at $6.27 \mathrm{MHz}\left(\mathrm{S}, 2 \mathrm{H}, \mathrm{NH}_{2}\right), 3.83 \mathrm{MHz}$ $\left(\mathrm{S}, 3 \mathrm{H}, \mathrm{CH}_{3} 23\right), 7.74 \mathrm{MHz}(\mathrm{d}, 1 \mathrm{H}, \mathrm{J}=6.4 \mathrm{~Hz}, \mathrm{H}-13), 7.78$ $\mathrm{MHz}(\mathrm{d}, 1 \mathrm{H}, \mathrm{J}=6.4 \mathrm{~Hz}, \mathrm{H}-9), 7.21 \mathrm{MHz}(\mathrm{d}, 1 \mathrm{H}, \mathrm{J}=1.2 \mathrm{~Hz}$, H-6), $6.90 \mathrm{MHz}(\mathrm{S}, 1 \mathrm{H}, \mathrm{H}-8), 8.06 \mathrm{MHz}$ (S, 2H, H-19,21), $7.35 \mathrm{MHz}(\mathrm{d}, 2 \mathrm{H}, \mathrm{J}=6.4 \mathrm{~Hz}, \mathrm{H}-15), 8.09 \mathrm{MHz}(\mathrm{d}, 2 \mathrm{H}, \mathrm{J}=6.4$ $\mathrm{Hz}, \mathrm{H}-16), 8.50 \mathrm{MHz}(\mathrm{d}, 2 \mathrm{H}, \mathrm{J}=6.8 \mathrm{~Hz}, \mathrm{H}-18,22), 7.41 \mathrm{MHz}$ (S, 1H, H-1), 5.35 MHz (S, 1H, O-H) (Fig. 2).
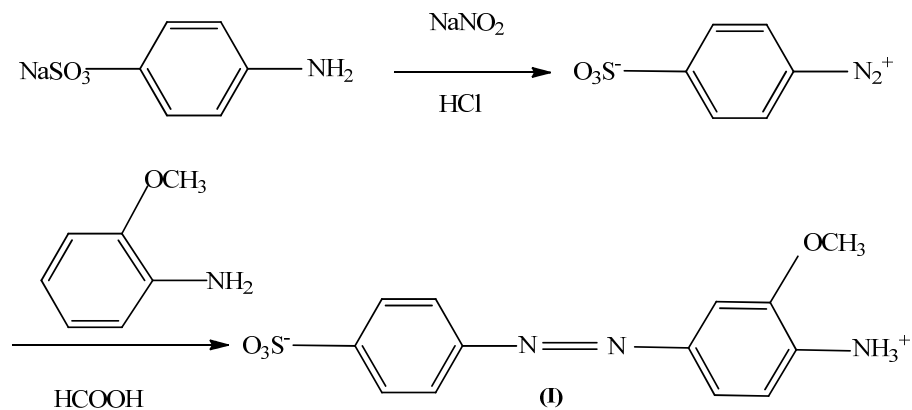

$\mathrm{HCOOH}$

(I)<smiles>CC(=O)O</smiles><smiles>COc1cc(/N=N/c2ccc(S(N)(=O)=O)cc2)ccc1/N=N/c1c(S(N)(=O)=O)cc2ccc(N)cc2c1O</smiles>

Fig. 1 - Synthesis of the dye A. 
<smiles>COc1cc(/N=N/c2ccc(OS(=O)(=O)O)cc2)ccc1/N=N/c1c([N+](=O)[O-])cc2ccc(N)cc2c1O</smiles><smiles>COc1cc(/N=C/c2ccc(S(=O)(=O)OC)cc2)ccc1/N=C/c1c([As])cc2ccc(N)cc2c1[AsH3]</smiles>

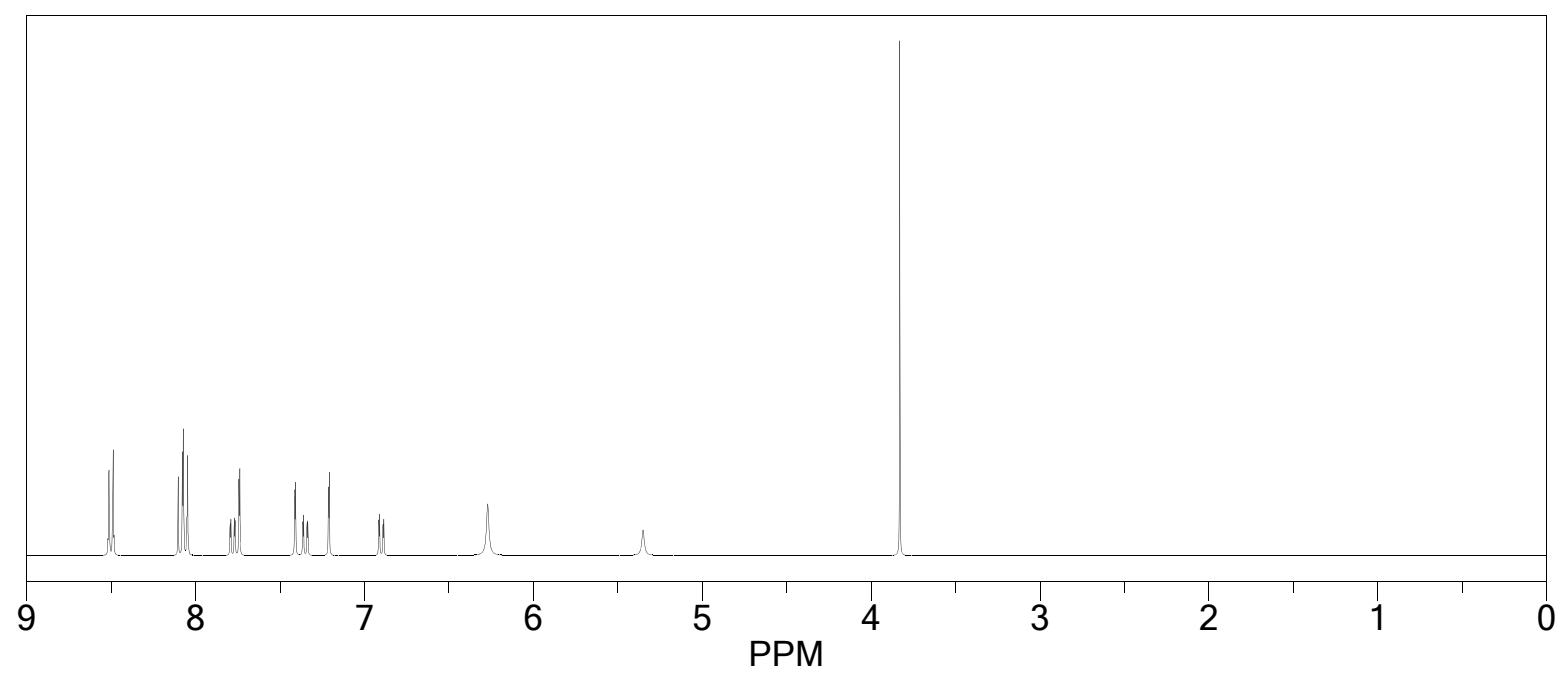

Fig. 2 - Experimental ${ }^{1} \mathrm{H}$ NMR Spectrum of the dye A.

Experimental FT-IR Spectrum of the dye A

$451.06 \mathrm{~cm}^{-1}$ bending vibrations of $=\mathrm{C}-\mathrm{H}$ aromatic, $530.08 \mathrm{~cm}^{-1}$ stretching vibrations $-\mathrm{NH}, 570.58 \mathrm{~cm}^{-1}$ out of plane aromatic ring vibrations, $630.43 \mathrm{~cm}^{-1}$ stretching vibrations $\mathrm{S}-\mathrm{O}, 705.89 \mathrm{~cm}^{-1} \mathrm{C}-\mathrm{H}$ bending vibrations $-\mathrm{NH}$, $1007.40 \mathrm{~cm}^{-1}$ stretching vibrations $\mathrm{C}=\mathrm{C}, 1035.38 \mathrm{~cm}^{-1}$ stretching vibrations $\mathrm{C}-\mathrm{N}, 1052.12 \mathrm{~cm}^{-1}$ stretching vibrations $\mathrm{S}=\mathrm{O}, 1122.17 \mathrm{~cm}^{-1}$ stretching vibration of $\mathrm{C}-\mathrm{O}, 1155.04 \mathrm{~cm}^{-1}$ stretching vibration of $\mathrm{C}-\mathrm{N}, 1402.08 \mathrm{~cm}^{-1}$ stretching vibration of $\mathrm{CH}_{3}, 1496.50 \mathrm{~cm}^{-1}$ stretching vibration of $\mathrm{N}=\mathrm{N}, 1631.07 \mathrm{~cm}^{-1}$ stretching vibration of $\mathrm{C}=\mathrm{C}$ aromatic, $1657.73 \mathrm{~cm}^{-1}$ stretching vibrations of $\mathrm{C}=\mathrm{C}$ aromatic (Fig. 3).

\section{Preparation of PVA-films containing the dye A}

The PVA-films were prepared from $10 \%$ PVA solution, containing the $0.01-0.05$ wt. $\%$ the dye A, 0.01 wt. \% boric acid $\left(\mathrm{H}_{3} \mathrm{BO}_{3}\right), 6.0$ wt. \% ethyl alcohol $\left(\mathrm{C}_{2} \mathrm{H}_{5} \mathrm{OH}\right)$ and water. An initial composition was prepared by dissolving PVA in distilled water and ethyl alcohol. The composition was mixed at temperature $85-90{ }^{\circ} \mathrm{C}$. The dye $\mathbf{A}$ and additives were added after 3 hours after starting of heating PVA solution at intervals of 20 minutes. The mixture was heated for 3 hours. The hot solution was filtered through two layers of technical nylon. Deaeration was occurred during 12-15 hours. The composition was cast on the polished glasses and dried in the closed box at temperature $20-22{ }^{\circ} \mathrm{C}$. Uniaxial orientation was done in the $4 \%$ boric acid solution at temperature $42-45{ }^{\circ} \mathrm{C}$. The washed film was dried for 30 minutes at temperature 60 $63{ }^{\circ} \mathrm{C}$. The value of $\mathrm{R}_{\mathrm{s}}$ was determined as the ratio between length of the films after and before $\left(l_{\text {aft }} / l_{\text {bef }}\right)$ uniaxial orientation. The thickness of the resulting films was between 50 to $55 \mu \mathrm{m} .{ }^{1-4}$ The film thickness was measured with a micrometer with an accuracy of $\pm 5 \mu \mathrm{m}$ (GS SSSR 6507-90). 
Accuracy of measurements in this research limits the accuracy of the results ${ }^{1}$ :

- Sample of the starting composition for preparation of solutions and films for casting taken on an analytical balance accurate to $0.0005 \mathrm{~g}$;

- Temperature measurement error was:

$\pm 2.0^{\circ} \mathrm{C}$ in the preparation of polymer solutions with additives,

$\pm 1.0^{\circ} \mathrm{C}$ in the chemical treatment of the films,

$\pm 3.0^{\circ} \mathrm{C}$ at thermal fixation and drying films;

- Relative error of spectrophotometric analysis did not exceed $1.0 \%$ and thermal conductivity $0.1 \%$.

The standard deviation of parallel measurements determined by the formula ${ }^{3}$ :

$$
S(d)=\sqrt{\sum_{i}\left(d_{i}-d\right)^{2} / n-1}
$$

where $d_{i}-$ the result of a single measurement, $\mathrm{d}$ - Arithmetic mean

$\mathrm{n}$ - Number of measurements.

\section{RESULTS AND DISCUSSION}

\section{Optical properties}

\section{Polarization of PVA-films containing the dye A}

The main optical properties of polarizing films such as Transmittance $\left(\mathrm{T}_{\perp}, \mathrm{T}_{\|}\right)$and Polarizing Efficiency (PE) were evaluated at the absorption maximum of the polarizing films according to Eqs. $(1)^{1-3}$ :

$$
\mathrm{PE}=\left(\mathrm{T}_{\perp}-\mathrm{T}_{\|}\right) /\left(\mathrm{T}_{\perp}+\mathrm{T}_{\|}\right) * 100
$$

where, $\mathrm{T}_{\|}, \mathrm{T}_{\perp}-$ Transmittance for linearly polarized light parallel $(\|)$ and perpendicular $\left({ }_{\perp}\right)$ to direction of stretching of colored film. Polarizing Efficiency of colored oriented PVA-films depends on the concentration of injected dye and Stretching Degree of the film, ${ }^{2-4}$ therefore the optimum concentration of the dye $\mathbf{A}$ in PVA-film was obtained (Table 1). Changes in concentration of the dye A from 0.10 to $0.50 \mathrm{wt}$. $\%$ in the colored oriented PVA-film show that with increasing concentration of the dichroic agent, maximum light transmission in parallel and perpendicular direction are reduced. And at $[\mathbf{A}]=0.18$ wt. $\%, \mathrm{PE}=91 \%$. With increasing concentration of the dichroic agent $(0.50$ wt. \%) PE decreases. It is due to the increasing intensity of the absorption of light by the dye.

Dichroism (anisotropy of absorption) (Fig. 4) appears under uniaxial orientation of the colored PVA-films (Fig. 4b).
We have studied effect of $R_{s}$ on PE of the PVAfilms (Table 2). Stretching (uniaxial orientation) of film containing $0.18 \mathrm{wt}$ \% of the dye $\mathbf{A}$ from 2.5 to 4.5 times leads to increase in its $\mathrm{T}_{\perp}$ from $31.6 \%$ to $42.0 \%$ and decrease PE from 92 to $85 \%$.

Thus we have found that a PVA-films containing 0.18 wt. $\%$ of the dye $\mathbf{A}$ at $\mathrm{R}_{\mathrm{s}}=2.5$ and 3 times have the best optical properties ( $\mathrm{PE}=92 \%$, $\mathrm{T}_{\perp}=31.6 \%, \mathrm{~T}_{\|}=1.3 \%$ and $\mathrm{PE}=92 \%, \mathrm{~T}_{\perp}=32.8 \%$, $\left.\mathrm{T}_{\|}=1.4 \%\right)($ Tables 1,2$)$.

\section{Absorption spectrum and electronic structure of the dye $A$}

Theoretical absorption spectrum of the molecule A optimized in a solvent (Water) was calculated using TDB3LYP/6-311++G** method at $300-700 \mathrm{~nm}$ by the IEFPCM solvent model with Water as the solvent. All the DFT computational calculations were carried out with the Gaussian $09 \mathrm{~W}$ package. ${ }^{18}$ For visualization of UV/Vis spectrum of the dye A Chemcraft software was used. ${ }^{19}$ The equations were solved for 10 excited states, where the computational studies were performed in presence Water as solvent, using the IEFPCM (integral equation formalism PCM) method coupled to UAKS radii. The IEF method originally developed by Cances and Mennuci introduces one function of permittivity dependent of the position, with this change. ${ }^{20}$ The TDDFT method is able to detect accurate absorption wavelength at a relatively small computing time, which corresponds to electronic transitions computed on the ground state geometry (Table 3). The experimental absorption spectrum of the dye A (Fig. 5 a) has a wide absorption at about 300$620 \mathrm{~nm}$ which characterized by maxima at 440 460 and $555 \mathrm{~nm}$ and calculated spectrum (Fig. $5 \mathrm{~b}$ ) shows transitions in 456 and $555 \mathrm{~nm}$ that the strongest is at $555 \mathrm{~nm}(\mathrm{f}=2.00)$. The strong peak at $555 \mathrm{~nm}$ is due to the Charge-Transfer (CT) excited state. The other peaks are local excited states corresponding to electrons going into antibonding orbitals associated with the benzene rings. Excitation of one electron at $555 \mathrm{~nm}$ belonged to the transition into the excited singlet state $S_{1}$ and describes by a wave function corresponding to a superposition of two configurations for oneelectron excitations $(134 \rightarrow 138$, and $134 \rightarrow 147)$. Excitation of an electron from 134 to 138 molecular orbital (MO) gives the main contribution to the formation of the absorption band at $555 \mathrm{~nm}$ (Fig. 6). Excitation of one electron at $456 \mathrm{~nm}$ 
belonged to the transition into the excited singlet state $S_{2}$ and describes by a wave function corresponding to a superposition of twelve configurations for one-electron excitations $(112 \rightarrow 136, \quad 112 \rightarrow 144, \quad 118 \rightarrow 135, \quad 118 \rightarrow 136$, $118 \rightarrow 144, \quad 127 \rightarrow 135, \quad 127 \rightarrow 136, \quad 127 \rightarrow 140$, $127 \rightarrow 144,129 \rightarrow 135,129 \rightarrow 136$ and $129 \rightarrow 144$ ). Excitation of an electron from 127 to 144 molecular orbital (MO) gives the main contribution to the formation of the absorption band at $456 \mathrm{~nm}$ (Table 3). The next strong absorption at $453 \mathrm{~nm}$ belonged to the transition into the excited singlet state $S_{3}$ and describes by a wave function corresponding to a superposition of six configurations for one-electron excitations $(128 \rightarrow 135$, $132 \rightarrow 135, \quad 133 \rightarrow 135,133 \rightarrow 136,133 \rightarrow 140$ and $134 \rightarrow 135)$. Excitation of an electron from 132 to 135 and 133 to $135 \mathrm{MO}$ gives the main contribution to the formation of the absorption band at $453 \mathrm{~nm}$ (Table 3, Fig. 5). The next absorption peak at $440 \mathrm{~nm}$ belonged to the transition into the excited singlet state $S_{4}$ and describes by a wave function corresponding to a superposition of five configurations for one-electron excitations $(131 \rightarrow 138,131 \rightarrow 147,134 \rightarrow 136,134 \rightarrow 139$ and $134 \rightarrow 140)$. Excitation of an electron from 134 to $139 \mathrm{MO}$ gives the main contribution to the formation of the absorption band at $440 \mathrm{~nm}$. Excited state $S_{5}$ at $416 \mathrm{~nm}$ describes by a wave function corresponding to a superposition of three configurations for one-electron excitations $(134 \rightarrow 138,134 \rightarrow 142$ and $134 \rightarrow 143)$. Excitation of an electron from 134 to $142 \mathrm{MO}$ gives the main contribution to the formation of the absorption band at $416 \mathrm{~nm}$ (Table 3, Fig. 5). Excited states in VU region of spectrum $\left(\mathrm{S}_{6} \div \mathrm{S}_{10}\right)$ are presented in Table 3 but not discussed.

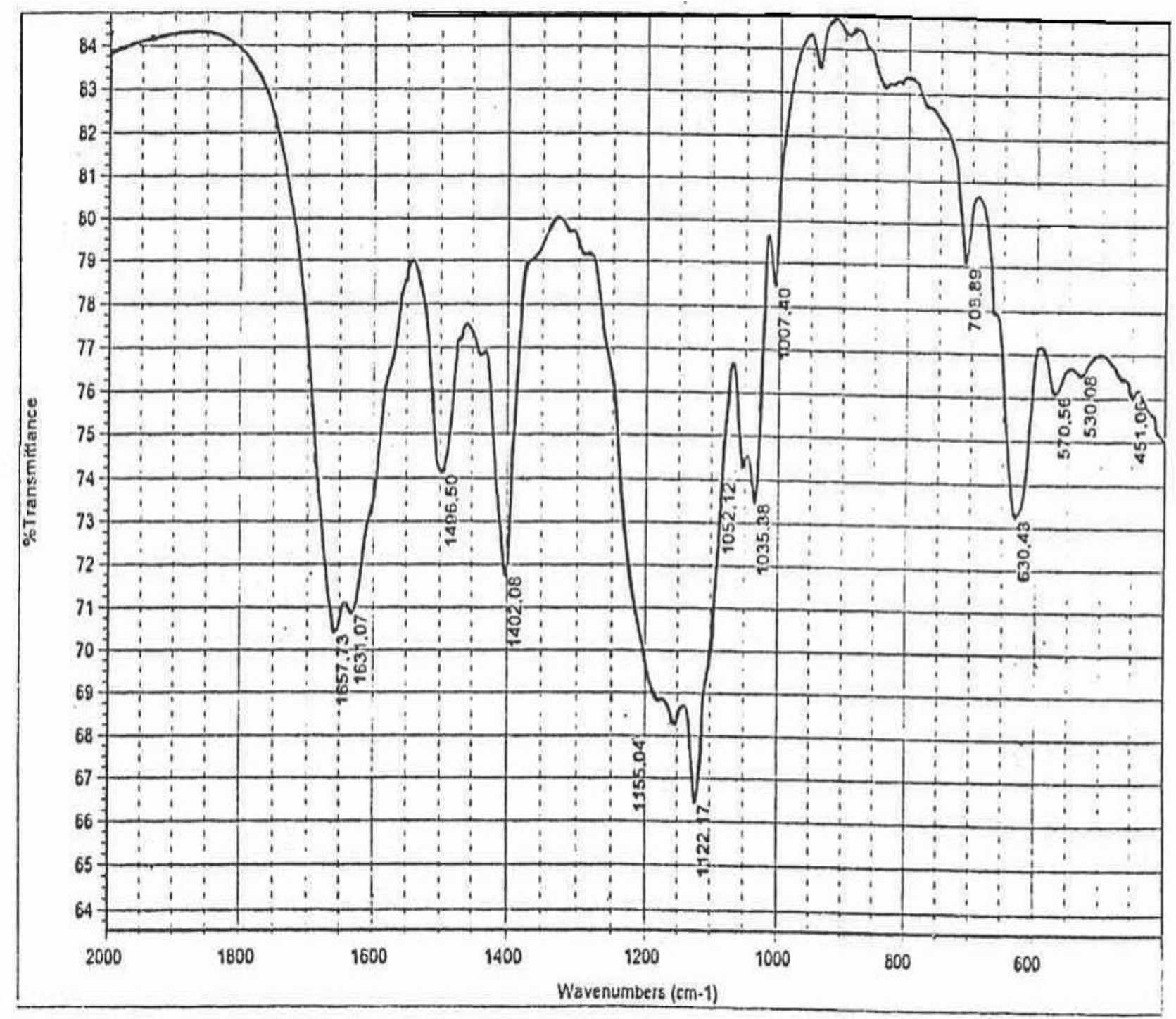

Fig. 3 - FT-IR spectrum of the dye A. 
Table 1

The optical characteristics of PVA-films at different concentration of the dye $\mathbf{A}, \mathrm{R}_{\mathrm{s}}=3.5$

\begin{tabular}{|c|c|c|c|}
\hline \multirow{2}{*}{$\begin{array}{c}\text { [A] in film, } \\
\text { wt. } \%\end{array}$} & \multicolumn{2}{|c|}{ Transmittance at $\lambda=550 \mathrm{~nm}, \%$} & \multirow[t]{2}{*}{ PE, \% } \\
\hline & $\mathbf{T}_{\perp}$ & $\mathbf{T}_{\|}$ & \\
\hline 0 & 91.9 & 90.6 & 0 \\
\hline 0.10 & 66.9 & 18.8 & 56 \\
\hline 0.14 & 56.6 & 10.1 & 70 \\
\hline 0.18 & 34.8 & 1.6 & 91 \\
\hline 0.50 & 15.3 & 1.0 & 88 \\
\hline
\end{tabular}

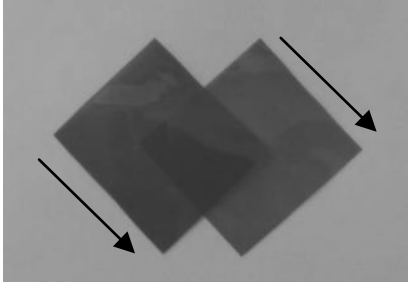

a

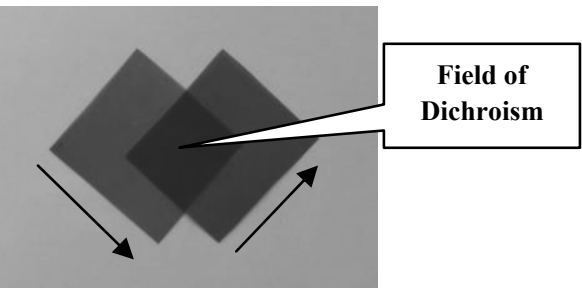

$\mathrm{b}$

Fig. 4 - PVA-films containing the dye $\mathbf{A}$ in parallel (a) and perpendicular (b) directions of stretching.

Table 2

Influence of $\mathrm{R}_{\mathrm{s}}$ on the optical characteristics of films containing [A] $=0.18 \mathrm{wt} . \%$

\begin{tabular}{c|c|c|c}
\hline \multirow{2}{*}{$\mathbf{R}_{\mathbf{s}}$} & \multicolumn{2}{|c|}{ Transmittance at $\boldsymbol{\lambda = \mathbf { 5 5 0 } \mathbf { ~ n m } , \mathbf { \% }}$} & \multirow{2}{*}{$\begin{array}{c}\text { PE, } \\
\mathbf{\%}\end{array}$} \\
\cline { 2 - 4 } & $\mathbf{T}_{\perp}$ & $\mathbf{T}_{\|}$ & 92 \\
3.5 & 31.6 & 1.3 & 92 \\
3.5 & 32.8 & 1.4 & 91 \\
4.0 & 34.8 & 1.6 & 88 \\
4.5 & 38.1 & 2.5 & 85 \\
\hline
\end{tabular}

Table 3

Electronic absorption spectrum of the compound A calculated by TDB3LYP/6-311++G** method

\begin{tabular}{|c|c|c|c|c|}
\hline $\begin{array}{l}\text { Excited } \\
\text { state }\end{array}$ & $\begin{array}{l}\text { Wavelength } \\
\text { (nm) }\end{array}$ & $\begin{array}{c}\text { Excitation } \\
\text { energy } \\
(\mathrm{eV})\end{array}$ & Configurations composition (corresponding transition orbitals) & $\begin{array}{l}\text { Oscillator } \\
\text { strength } \\
\text { (f) }\end{array}$ \\
\hline $\mathrm{S}_{1}$ & 555 & 2.23 & $0.59(134 \rightarrow 138)-0.28(134 \rightarrow 147)$ & 2.00 \\
\hline $\mathrm{S}_{2}$ & 456 & 2.72 & $\begin{array}{l}0.11(112 \rightarrow 136)+0.11(112 \rightarrow 144)-0.19(118 \rightarrow 135) \\
+0.22(118 \rightarrow 136)+0.24(118 \rightarrow 144)+0.19(127 \rightarrow 135) \\
-0.19(127 \rightarrow 136)+0.11(127 \rightarrow 140)-0.27(127 \rightarrow 144) \\
+0.15(129 \rightarrow 135)-0.14(129 \rightarrow 136)-0.22(129 \rightarrow 144)\end{array}$ & 0.80 \\
\hline $\mathrm{S}_{3}$ & 453 & 2.74 & $\begin{array}{l}0.16(128 \rightarrow 135)+0.35(132 \rightarrow 135)+0.35(133 \rightarrow 135) \\
+0.17(133 \rightarrow 136)-0.12(133 \rightarrow 140)-0.15(134 \rightarrow 135)\end{array}$ & 0.78 \\
\hline $\mathrm{S}_{4}$ & 440 & 2.82 & $\begin{array}{c}0.17(131 \rightarrow 138)-0.16(131 \rightarrow 147)+0.13(134 \rightarrow 136) \\
\quad+0.45(134 \rightarrow 139)+0.44(134 \rightarrow 140)\end{array}$ & 0.68 \\
\hline $\mathrm{S}_{5}$ & 416 & 2.98 & $0.14(134 \rightarrow 138)+0.58(134 \rightarrow 142)-0.30(134 \rightarrow 143)$ & 0.50 \\
\hline $\mathrm{S}_{6}$ & 379 & 3.27 & $\begin{array}{c}-0.17(128 \rightarrow 135)-0.25(130 \rightarrow 135)-0.20(132 \rightarrow 135) \\
+0.12(132 \rightarrow 136)+0.13(132 \rightarrow 139)-0.13(132 \rightarrow 140) \\
+0.21(133 \rightarrow 135)+0.18(133 \rightarrow 136)-0.16(133 \rightarrow 140) \\
-0.21(134 \rightarrow 145)\end{array}$ & 0.24 \\
\hline $\mathrm{S}_{7}$ & 356 & 3.48 & $\begin{array}{c}0.18(127 \rightarrow 137)-0.11(127 \rightarrow 141)-0.27(127 \rightarrow 146) \\
+0.25(128 \rightarrow 135)-0.18(128 \rightarrow 136)-0.10(128 \rightarrow 144) \\
-0.18(130 \rightarrow 135)+0.26(130 \rightarrow 136)+0.19(130 \rightarrow 144) \\
+0.14(132 \rightarrow 136)+0.14(132 \rightarrow 144)\end{array}$ & 0.27 \\
\hline $\mathrm{S}_{8}$ & 331 & 3.75 & $\begin{array}{c}-0.11(128 \rightarrow 135)-0.11(133 \rightarrow 135)-0.42(134 \rightarrow 135) \\
+0.24(134 \rightarrow 136)+0.20(134 \rightarrow 139)-0.27(134 \rightarrow 140) \\
-0.15(134 \rightarrow 144)+0.13(134 \rightarrow 150)\end{array}$ & 0.06 \\
\hline
\end{tabular}


Table 3 (continued)

\begin{tabular}{|c|c|c|c|c|}
\hline $\mathrm{S}_{9}$ & 326 & 3.80 & $\begin{array}{c}-0.14(123 \rightarrow 135)+0.11(127 \rightarrow 137)-0.11(128 \rightarrow 136) \\
-0.12(129 \rightarrow 146)+0.32(131 \rightarrow 138)-0.14(131 \rightarrow 142) \\
-0.26(131 \rightarrow 147)-0.13(133 \rightarrow 135)-0.12(134 \rightarrow 139) \\
-0.11(134 \rightarrow 140)-0.27(134 \rightarrow 145)\end{array}$ & 0.49 \\
\hline $\mathrm{S}_{10}$ & 311 & 3.99 & $\begin{array}{c}-0.12(119 \rightarrow 143)-0.10(122 \rightarrow 135)+0.10(125 \rightarrow 135) \\
+0.10(125 \rightarrow 136)-0.11(130 \rightarrow 135)+0.11(132 \rightarrow 135) \\
+0.11(132 \rightarrow 139)+0.46(134 \rightarrow 145)\end{array}$ & 0.14 \\
\hline
\end{tabular}
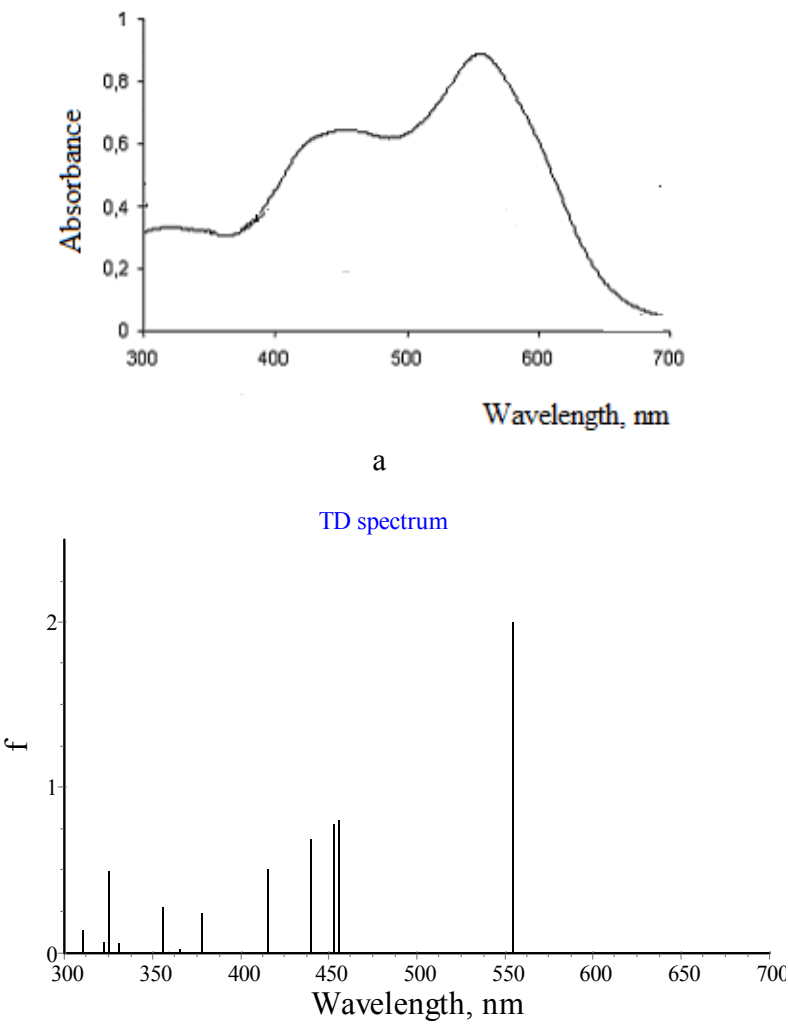

$\mathrm{b}$

Fig. 5 - UV-Vis Spectrum of the dye A in Water: (a) experimental at dye concentration $3.6 \cdot 10^{-4} \mathrm{M} / \mathrm{L}$ and (b) calculated by TDB3LYP/6-311++G** method.

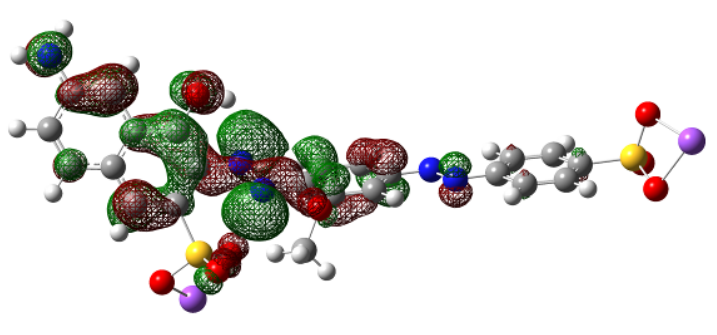

$134 \mathrm{MO}$

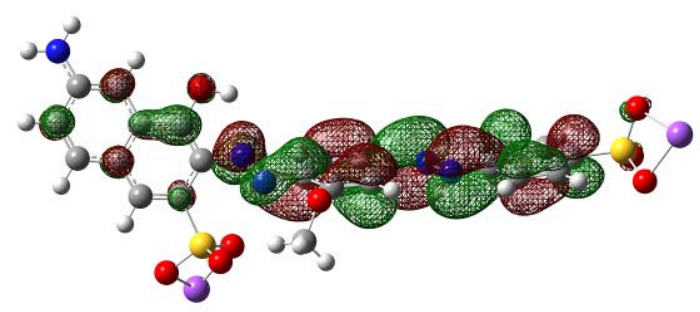

$138 \mathrm{MO}$

Fig. 6 - Form of the MO involved in formation of absorption spectrum of the compound at $\lambda_{\max }=555 \mathrm{~nm}$ calculated by B3LYP/6-311++G** method.

\section{Thermo-physical properties}

To clarify the nature of the effect of dyes on thermal stability of polyvinyl alcohol matrix, thermal conductivity of colored films has been studied. Experimental data on the thermal properties of an anisotropic medium allow estimating the role of structural parameters and the 
strength of bond between molecules and their aggregates in the process of heat transfer. In polymeric substances covalent bond generally predominates, and the motion of atoms in a polymer substance appears as collective oscillations of the atoms of the entire system. ${ }^{2}$ Heating polymer increases the amplitude of the oscillations of the atoms. Heat is transferred through the interatomic forces from the hotter region to less heated one until all parts of the body acquire one common temperature. Besides the thermal motion of molecule segmental areas which appears at the glass transition temperature of the polymer, movement of individual fragments or monomeric units is possible. ${ }^{2,3}$ One should also distinguish heat transfers inside a macromolecule and between molecules. It is established ${ }^{3}$ that the thermal fluctuations in the macromolecule are transmitted much faster on the main valences than on (auxiliary ones) the side. Intermolecular heat transfer in certain PVA is characterized by the existence of a potential barrier (or thermal resistance) in the heat pulse transfer caused by Van der Waals forces of interaction. As a result, the thermal conductivity $(\lambda)$ in this direction is characterized by smaller values. It is known ${ }^{2}$ that fillers and modifiers of plastics material modify the thermal conductivity of polymers. The degree of influence depends on the size and shape of the particles, their distribution in the polymer matrix and interaction of filler or a modifier with a polymer. ${ }^{3}$ Consequently, coloring materials as modifiers of the film properties influence their heat conductivity. There are various types of polymer polarizers, but the ones that are mostly used in the practice are obtained on the basis of PVA, colored with iodine or organic dyes such as azo benzene derivatives, which are more resistant to high temperatures and humidity of the environment. The search and synthesis of new effective dichroic dyes remains an urgent task of research in the field of thermostable polarizing films. ${ }^{2,3,5}$

\section{Anisotropy of thermal conductivity of PVA-films}

We have studied thermal conductivity of the systems: PVA-A for development of thermostable polarizing films. In the researches were used special paints able to sharply change the initial color at a critical temperature $\left(\mathrm{T}_{\text {cr }}\right)$. The isothermal surface moves at a certain speed in the proportion to a local gradient of the temperature field. Thermo-physical characteristics of material can be judged by the speed of isotherm movement and the form of flowed surface. Thermal indicators of Ciba Com. (Switzerland) with small sizes of $\mathrm{T}_{\mathrm{cr}}$ are used in order to avoid thermal destruction of polymer materials. Thermal indicator is put on a film by a thin layer and at regular intervals by a draw plate. Then, after some drying period its unpainted surface is resulted on some time $(30 \mathrm{sec}$.) in dense contact to a dot source of heat (it is heated up approximately to $55{ }^{\circ} \mathrm{C}$ metal needles). For estimation of thermo-physical properties of films, heat conductivity of samples was determined in parallel $\left(\lambda_{\|}\right)$and perpendicular $\left(\lambda_{\perp}\right)$ directions of stretching axis. During this work it was established that the oriented PVA-films with the dye A is the phenomenon of anisotropy of thermal conductivity $\left(\lambda_{\|} / \lambda_{\perp}\right)$. Anisotropy of thermal conductivity in unstretched PVA-films is observed not appreciably $\left(\lambda_{\|}=0.875 \mathrm{~W} / \mathrm{m} .{ }^{\circ} \mathrm{C} ; \lambda_{\perp}=0.869 \mathrm{~W} / \mathrm{m} .{ }^{\circ} \mathrm{C}\right)$ as comparison to stretched PVA-films that is observed very clearly (Table 4).

Results of thermal conductivity measurements of PVA-films containing the dye A depending on stretching degree are given in Table 5. It is clear from the results that the thermal conductivity in a direction $\left(\lambda_{\|}\right)$orientation is higher than perpendicular orientations $\left(\lambda_{\perp}\right)$. On resulting anisotropy at a known degree of extension it is possible to judge anisotropy of chain structure. It is necessary to notice that during thermal expansion and thermal conductivity, geometric parameters of molecule and intermolecular forces play a significant role.

Table 4

Dependence of thermal conductivity on stretching degree in pure PVA-films

\begin{tabular}{c|c|c|c}
\hline \multirow{2}{*}{$\mathbf{R}_{\mathbf{s}}$} & \multicolumn{2}{|c}{$\boldsymbol{\lambda}$} \\
\cline { 2 - 4 } & $\boldsymbol{\lambda}_{\|}, \mathbf{W} / \mathbf{m} .^{\mathbf{0}} \mathbf{C}$ & $\boldsymbol{\lambda}_{\perp}, \mathbf{W} / \mathbf{m} .^{{ }^{\circ}} \mathbf{C}$ & $\lambda_{\|} / \boldsymbol{\lambda}_{\perp}$ \\
\hline 1.5 & 0.876 & 0.764 & 1.15 \\
2.0 & 0.878 & 0.636 & 1.38 \\
2.5 & 0.880 & 0.549 & 1.60 \\
3.0 & 0.881 & 0.503 & 1.75 \\
4.0 & 0.882 & 0.475 & 1.86 \\
\hline
\end{tabular}


Table 5

Thermal conductivity of PVA-films containing the dye A at concentration 0.18 wt. $\%$

\begin{tabular}{c|c|c|c}
\hline \multirow{2}{*}{$\mathbf{R}_{\mathbf{s}}$} & \multicolumn{3}{|c}{$\boldsymbol{\lambda ,}$} \\
\cline { 2 - 4 } & $\lambda_{\|}, \mathbf{W} / \mathbf{m} .^{\circ} \mathbf{C}$ & $\lambda_{\perp}, \mathbf{W} / \mathbf{m} .^{\circ} \mathbf{C}$ & $\lambda_{\|} / \lambda_{\perp}$ \\
3.0 & 0.865 & 0.261 & 3.31 \\
4.0 & 0.851 & 0.247 & 3.44 \\
5.0 & 0.844 & 0.186 & 4.54 \\
\hline
\end{tabular}

The occurrence of anisotropy of thermal conductivity is connected that at orientation of PVA occurs orientation of the amorphous part of polymer and also formation of additional number of intermolecular connections. Thermal conductivity of PVA films changes after the injection of dye (Table 5) and along an axis of orientation and in perpendicular axis decreases.

\section{CONCLUSIONS}

In the present work, we have calculated the electronic structure and UV/Vis spectrum of the molecule: Sodium 6-amino-5-hydroxy-3-((E)(2-methoxy-4-((E)-(4-sulfonatophenyl) diazenyl) phenyl)diazenyl)naphthalene-2-sulfonate (A) by using B3LYP/6-311++G** method. Based on quantum-chemical calculations have been synthesized new azo benzene dichroic dye for visible region of spectrum (at 440-460 and $550 \mathrm{~nm}$ ). At the first time on the basis of polyvinyl alcohol (PVA) and synthesized compound: Sodium 6-amino-5-hydroxy-3-((E)-(2-methoxy-4-((E)-(4sulfonatophenyl)diazenyl)phenyl)diazenyl) naphthal ene-2-sulfonate (A) thermostable polarizing film absorbing at $\lambda_{\max }=550 \mathrm{~nm}$ was developed. Stretched colored PVA-films have phenomenon of anisotropy of thermal conductivity. Thermal conductivity in a direction of orientation $\left(\lambda_{\|}\right)$is higher than in a direction perpendicular orientations $\left(\lambda_{\perp}\right)$. In stretched dyed PVA-films anisotropy of thermal conductivity is observed very clearly (at $\left.R_{s}=5.0, \lambda_{\|} / \lambda_{\perp}=7.36\right)$.

\section{REFERENCES}

1. V. Agabekov, A. Muravsky, N. Ariko, S. Shahab and A. J. Tolstic, Semicond. Phys. Quantum. Electro. Optoelectro., 2010, 13, 32-35.

2. S. Shahab, V. Agabekov, N. Ariko and L. Filippovich, Anisotropy of polarizing polyvinyl alcohol films,
Advanced Display Technologies: Proc. of the 15 Intern. Symp., Moscow, Russia, Oct. 3-5, 2006 / Soc. for Inform. Display. 163-166.

3. V. Agabekov, S. Shahab, N. Ariko and L. Filippovich, Anisotropy of polarizing films with azodyes, $X V$ Intern. Symp. On Advanced Dis. Tech. SPIE., San Diego, USA, August 27-31, 2007. V. 19/4, 174-179.

4. V. Agabekov, N. Ivanova, S. Shahab, I. Kulevskaya and N. Ariko, Film transfect polarizer, Advanced Display Technologies: Basic Studies of Problems in Inform. Display (FLOWERS 2000), Moscow, Russia, Oct. 1012, 2000. / ed. V.V. Belyaev, I.N. Kompanets; Soc. for Inform. Display. - Bellingham, 2000, 141-143.

5. H. A. Almodarresiyeh, S. N. Shahab,V. M. Zelenkovsky and V. E. Agabekov, J. App. Spect., 2014, 81, 181-183.

6. H. A. Almodarresiyeh, S. N. Shahab, V. M. Zelenkovsky, N. G. Ariko, L. N. Filippovich and V. E. Agabekov, J. App. Spect., 2014, 81, 42-47.

7. O. Yaroschuk, T. Sergan, J. Lindau, S. Lee, J. Kelly and L. C. Chien, J. Chem. Phys., 2001, 114, 5330-5337.

8. J. J. Kim., K. Funabiki, H. Muramatsu, K. Shibata, S. H. Kim, H. Shiozaki, H. Hartmann and M. Matsui, J. Chem. Soci. Perkin Transactions, 2001, 2, 379-387.

9. C. Oprea, F. Moscalu, A. Dumbrava, S. Ioannou, A. Nicolaides and M. Girtu, Roman. J. phys., 2011, 56, 125-133.

10. E. M. Bezerra, J. R. Bezerra-Neto, F. A. Sales, R. P. dos Santos, A. Martins, P. de Lima-Neto, E. W. S. Caetano, E. L. Albuquerque and V. N. Freire, Molecules, 2014, 19, 4145-4156.

11. M. S. Masoud, A. E. Ali., M. A. Shaker and M. A. Ghani, Spectrochim. Acta Part A, 2004, 60, 3155-3159.

12. S. Shahab, L. Filippovich, R. Kumar, M. Darroudi, M. Yousefzadeh Borzehandani, M. Gomar and F. Haji Hajikolaee, J. Mol. Struct., 2015, 1101, 109-115.

13. S. Demukhamedova, I. Alieva, Z. Gadzhiev and N. Godzhaev, J. Qafqaz Univ. Chem., 2009, 27, 15-25.

14. S. Fleming, A. Mills and T. Tutte, J. Org. Chem., 2011, 7, 432-441.

15. P. M. Anbarasan, P. S. Kumar, K. Vasudevan, R. Govindan, A. Prakasam and M. Geetha, Eur. J. Chem., 2011, 2, 206-213.

16. S. Shahab, H. Alhosseini Almodarresiyeh, R. Kumar and M. Darroudi, J. Mol. Struct., 2015, 1088, 105-110.

17. S. Shahab, R. Kumar, M. Darroudi and M. Yousefzadeh Borzehandani, J. Mol. Struct., 2015, 1083, 198-203.

18. M. J. Frisch, Gaussian 09, Revision A.1 Gaussian Inc., Wallingford CT, 2009.

19. http://www.chemcraftprog.com/index.html

20. B. Mennucci, E. Cancés and J. Tomasi, J. Phys. Chem. B, 1997, 101, 10506-10517. 
